\title{
The Role of Surface and Interface Energy on Phase Stability of Nanosized Insertion Compounds
}

\author{
By Marnix Wagemaker,* Fokko M. Mulder, and Anton Van der Ven*
}

The exploration of nanoscale materials during the past decade has led to great advances in both sciences and technology. Recent developments in the field of nanoionics is offering new possibilities and challenges for the much required future energy-storage and conversion devices. ${ }^{[1,2]}$ When particle sizes approach the nanoscale, the influence of surfaces and interfaces can no longer be neglected in the rationalization and prediction of a material thermodynamic and kinetic properties. ${ }^{[3,4]}$ Recent observations in crystalline battery materials ${ }^{[5-9]}$ and hydrogenstorage materials ${ }^{[10-14]}$ that undergo a first-order phase transition upon ionic insertion indicate that a reduction in particle size causes a change of the equilibrium compositions, leading to a decrease or a shift of the miscibility gap. Surprisingly, such nanoscale effects already set in at particle sizes as large as 100 to $150 \mathrm{~nm}$. This has direct consequences for the voltage profile during battery charging or the equilibrium pressure during hydrogen loading, ${ }^{[6,9,15,16]}$ which for large particles are constant during a two-phase reaction. Generally, a reduced voltage or increased pressure plateau is observed in a two-phase reaction within nanoparticles, indicating a lower stability of the $\mathrm{Li} / \mathrm{H}$ rich phase. $^{[10,13-16]}$

Changes in thermodynamic properties at the nanoscale can also impact kinetic behavior. It has for example been proposed that increases in vacancy concentration due to altered equilibrium compositions at the nanoscale enhances ionic mobilities to render reversibility of hydrogen loading possible in metal hydrides ${ }^{[10,11]}$ and to enable Li-insertion in otherwise inactive electrode materials. ${ }^{[8,15]}$ Nanoscaling has also been identified as playing an important role in enhancing (dis)charge rates in $\mathrm{Li}_{x} \mathrm{FePO}_{4}$, as it seems to increase equilibrium compositions and thereby reduce the lattice mismatch between coexisting phases, which is responsible for large energy barriers to two-phase decomposition due to coherency strains. ${ }^{[9]}$

In this paper, we derive general equilibrium criteria for two-phase coexistence within insertion compound nanocrystallites, explicitly accounting for surface and interface free-energy contributions. For typical values of surface and interface energies estimated from first principles for $\mathrm{Li}_{x} \mathrm{FePO}_{4},{ }^{[17]}$ we find that

[*] Dr. M. Wagemaker, F. M. Mulder

Department of Radiation, Radionuclides and Reactors

Faculty of Applied Sciences

Delft University of Technology

Mekelweg 15, 2629 JB Delft (The Netherlands)

E-mail: m.wagemaker@tudelft.nl

Dr. A. Van der Ven

Department of Materials Science and Engineering

University of Michigan

Ann Arbor, MI 48109 (USA)

E-mail: avdv@umich.edu

DOI: 10.1002/adma.200803038 interface contributions in particular can have a pronounced effect on equilibrium compositions for two-phase coexistence in nanocrystallites. Not only do the solubility limits and equilibrium compositions depend on the particle size, they appear surprisingly sensitive to the particle shape, rationalizing recent inconsistent observations of solubility limits in nanosized $\mathrm{LiFePO}_{4}$. The derived equilibrium criteria for two-phase coexistence in nanocrystallites also shows that the equilibrium compositions are not constant, but depend on the overall concentration of the particle. This prediction implies that equilibrium compositions measured electrochemically (based on concentrations coinciding with the onset of two-phase coexistence) will not necessary produce the same values as those measured using diffraction techniques (such as neutron diffraction). We also provide simple graphical construction methods to assess the role of surface and interface free energies on two-phase coexistence within insertion compound crystallites.

We consider crystalline host materials that undergo a first-order phase transformation upon insertion of interstitial $\mathrm{Li}$ ions from a Li poor phase $(\alpha)$ to a Li rich phase $(\beta)$. We denote the bulk Gibbs free energies at constant temperature $(T)$ and pressure $(P)$ of the $\alpha$ and $\beta$ phases by $g_{\alpha}\left(x_{\alpha}\right)$ and $g_{\beta}\left(x_{\beta}\right)$,defined per interstitial site. The concentrations $x_{\alpha}$ and $x_{\beta}$ refer to the fraction of filled interstitial sites in the $\alpha$ and $\beta$ phases. For small crystallites, surface and interfacial free energies, $\sigma$ and $\gamma_{\alpha \beta}$, become important. If the crystallite consists of a single phase, its free energy can be written as

$G(x)=\frac{V}{\Omega} g_{\varepsilon}(x)+\sum_{i}^{\text {surf }} S_{i} \sigma_{\varepsilon}^{i}$

where $\varepsilon$ refers to either $\alpha$ or $\beta$ and $V$ and $\Omega$ correspond to the particle volume and volume per Li site, respectively (hence $V / \Omega$ is equal to the total number of interstitial sites). The $S_{i}$ are the surface areas for each surface $i$ having surface energy $\sigma^{i}$, and $x$ corresponds to the fraction of occupied interstitial sites of the homogeneous crystallite.

If a single crystallite consists of the two coexisting phases, $\alpha$ and $\beta$, the free energy of the crystallite will contain extra terms arising from the interface separating $\alpha$ and $\beta$

$$
\begin{aligned}
G= & \frac{V}{\Omega} \phi_{\alpha} g_{\alpha}\left(x_{\alpha}\right)+\frac{V}{\Omega} \phi_{\beta} g_{\beta}\left(x_{\beta}\right)+\sum_{i}^{\alpha \operatorname{Surf}} S_{\alpha}^{i} \sigma_{\alpha}^{i} \\
& +\sum_{j}^{\beta \operatorname{Surf}} S_{\beta}^{j} \sigma_{\beta}^{j}+\sum_{k}^{\alpha \beta \text { Int }} A_{\alpha \beta}^{k} \gamma_{\alpha \beta}^{k}
\end{aligned}
$$

where $\gamma_{\alpha \beta}^{k}$ is the interface free energy between $\alpha$ and $\beta$ for interface $k$, having area $A_{\alpha \beta}^{k} . \phi_{\alpha}$ and $\phi_{\beta}$ are the fraction of 
interstitial sites belonging to the $\alpha$ and $\beta$ phases, respectively, with $\phi_{\alpha}+\phi_{\beta}=1$ (phase fractions of $\alpha$ and $\beta$ ). The surface and interface areas, $S_{\alpha}^{i}, S_{\beta}^{j}$, and $A_{a \beta}^{k}$ as well as the volume per interstitial site, $\Omega$, are defined relative to the dimensions of the crystallite within a convenient reference state (the host without Li). Within this convention, the surface and interface free energies $\sigma_{\alpha}^{i}, \sigma_{\beta}^{j}$, and $\gamma_{\alpha \beta}^{k}$ are per unit area within the reference state. The summations run over all surfaces and interfaces of the crystallite with

$\sum^{\alpha \text { Surf }} S_{\alpha}^{i}+\sum^{\beta S u r f} S_{\beta}^{j}=S$, where $S$ is the total surface area of the crystallite within the reference state and is therefore constant. In Equation 2, we have neglected strain-energy contributions that arise in coherent equilibrium when $\alpha$ and $\beta$ have different equilibrium lattice parameters.

Equilibrium in a crystallite having concentration $x$ is determined by the state $(\alpha, \beta$, or $\alpha+\beta)$ having the lowest free energy. If at concentration $x$, Equation 2 is lower than Equation 1 (for $\varepsilon=\alpha$ or $\beta$ ) the crystallite will present two coexisting phases. It is common to define the composition above (below) which the $\alpha(\beta)$ phase becomes metastable relative to $\alpha+\beta$ two-phase coexistence as a solubility limit. We will denote the solubility limits by $x_{\alpha} *$ and $x_{\beta} *$. For nanosized crystallites where interface and surface free energies are important, it is also necessary to define a second set of concentrations, $x_{\alpha}$ and $x_{\beta}$, corresponding to the equilibrium concentrations in the $\alpha$ and $\beta$ phases when they coexist within the same crystallite. While in the large crystallite limit, these two sets of concentrations are equal to each other; we will show below that at the nanoscale the solubility limits $x_{\alpha}{ }^{*}$ and $x_{\beta}{ }^{*}$ are not equal to the concentrations $x_{\alpha}$ and $x_{\beta}$ of $\alpha$ and $\beta$ coexisting in the same crystallite.

To derive equilibrium criteria that determine the equilibrium concentrations within coexisting $\alpha$ and $\beta$ phases, we proceed as follows. At constant T, $P$, and $N$ (total amount of Li-ions in the system), a crystallite consisting of two coexisting phases $\alpha$ and $\beta$ will have two independent internal degrees of freedom that are not controlled by external boundary conditions (we assume a fixed geometry for the interface separating $\alpha$ from $\beta$ ). These are the number of Li ions residing in the $\alpha$ phase, $N_{\alpha}=N-N_{\beta}$, and the fraction of the crystallite that is in the $\alpha$ phase, $\phi_{\alpha}=1-\phi_{\beta}$ (alternatively, we could have chosen $N_{\beta}$ and $\phi_{\beta}$ as independent variables). In two-phase equilibrium at constant $T, P$, and $N$, the crystallite will choose values for $N_{\alpha}$ and $\phi_{\alpha}$ that minimize the total free energy in Equation 2. A necessary condition for this minimum in total free energy $G$ is that its partial derivatives with respect to $N_{\alpha}$ and $\phi_{\alpha}$ are zero,

$$
\left(\frac{\partial G}{\partial N_{\alpha}}\right)_{\varphi_{\alpha}}=0, \quad\left(\frac{\partial G}{\partial \varphi_{\alpha}}\right)_{N_{\alpha}}=0
$$

The dependence of Equation 2 on $N_{\alpha}$ can be made explicit by expressing the fraction of occupied interstitial sites $x_{\alpha}$ and $x_{\beta}$ as

$$
x_{\alpha}=\frac{\Omega N_{\alpha}}{\phi_{\alpha} V}, \quad x_{\beta}=\frac{\Omega\left(N-N_{\alpha}\right)}{\left(1-\phi_{\alpha}\right) V}
$$

The partial derivatives (Eq. 3) then yield two equilibrium criteria for two-phase coexistence in a small crystallite, $\mu_{\alpha}=\mu_{\beta}$

$$
\begin{aligned}
& \left(g_{\alpha}-x_{\alpha} \mu_{\alpha}\right)-\left(g_{\beta}-x_{\beta} \mu_{\beta}\right) \\
& +\frac{\Omega}{V}\left\{\sum_{i}^{\alpha \operatorname{Surf}} \frac{\partial S_{\alpha}^{i}}{\partial \varphi_{\alpha}} \Delta \sigma^{i}+\sum_{k}^{\alpha \beta \text { Int }} \frac{\partial A_{\alpha \beta}^{k}}{\partial \varphi_{\alpha}} \gamma_{\alpha \beta}^{k}\right\}=0
\end{aligned}
$$

In Equation 4, $\mu$ 's correspond to Li chemical potentials and are the slopes of $g$ with respect to $x: \mu=\partial g / \partial x$ (in contrast to a substitutional compound $\mathrm{Li}_{x} \mathrm{~K}_{1-x}$, where the chemical potential corresponds to the intercept of the common tangent with the composition axis). In Equation 5, $\Delta \sigma=\sigma_{\alpha}-\sigma_{\beta}$. The derivation of the equilibrium criteria Equations 4 and 5 assumes that the surface and interface free energies are independent of $\mathrm{Li}$ concentration. Furthermore, Equations 4 and 5 also rely on the assumption that the surface and interface excess Li concentrations and the surface and interface excess stresses are zero. Surface excess concentrations, for example, will modify the measured voltage profile for single-phase nanocrystallites compared with that of large crystallites. Surface excess stresses tend to increase the pressure within the crystallite in excess of the ambient pressure, even for particles with large flat surfaces.

For crystallites of macroscopic dimensions, $1 / \mathrm{V}$ approaches zero and the contribution from surfaces and interfaces in Equation 5 becomes negligible. In that limit, Equations 4 and 5 reduce to the familiar equilibrium criteria of two-phase coexistence within a crystalline host having a mobile interstitial component. The first criteria, Equation 4, requires that the chemical potentials of the interstitial component are equal in the coexisting phases, while Equation 5 with surface and interface terms neglected reduces to the equality of grand canonical potentials $\Phi=g-x \mu$ of the coexisting phases $\alpha$ and $\beta$. In a $g(x)$ versus $x$ diagram, Equations 4 and 5 in the large-crystallite limit is a mathematical formulation of the common tangent construction to graphically estimate the equilibrium compositions of the coexisting phases, $x_{\alpha}$ and $x_{\beta} \cdot{ }^{[18]}$ Indeed, Equation 4 requires that the tangents to $g_{\alpha}$ and $g_{\beta}$ at $x_{\alpha}$ and $x_{\beta}$, respectively, have the same slope, while Equation 5 in the large-particle limit requires that the intercept of the tangents to $g_{\alpha}$ and $g_{\beta}$ at $x_{\alpha}$ and $x_{\beta}$ with the $x=0$ axis, $\Phi_{\alpha}=g_{\alpha}-x_{\alpha} \mu_{\alpha}$ and $\Phi_{\beta}=g_{\beta}-x_{\beta} \mu_{\beta}$, be equal. As is well known, the equilibrium compositions, $x_{\alpha}$ and $x_{\beta}$, in the large-crystallite limit are independent of the phase fraction.

The terms involving surface and interface free energies can no longer be neglected in Equation 5 when crystallite dimensions approach the nanoscale. As the crystallite size decreases, the interface free energy penalty will become more important. The solubility limits $x_{\alpha}{ }^{*}\left(x_{\beta}{ }^{*}\right)$ should therefore increase (decrease) as crystallite dimensions are reduced. In this limit, the equilibrium compositions of the coexisting phases, $x_{\alpha}$ and $x_{\beta}$, become size and shape dependent. Furthermore, the differential changes in surface and interface area with phase fraction, $\varphi_{\alpha}$, will in general depend on the phase fraction. Hence, the equilibrium compositions $x_{\alpha}$ and $x_{\beta}$ are no longer necessarily constant, but can change with the overall Li composition of the crystallite.

Here we study the three basic particle geometries shown in Figure 1: a rectangular crystallite, a spherical crystallite, and a diamond-shaped crystallite. It will be assumed that $L_{x}=L_{z}=L_{x z}$ 


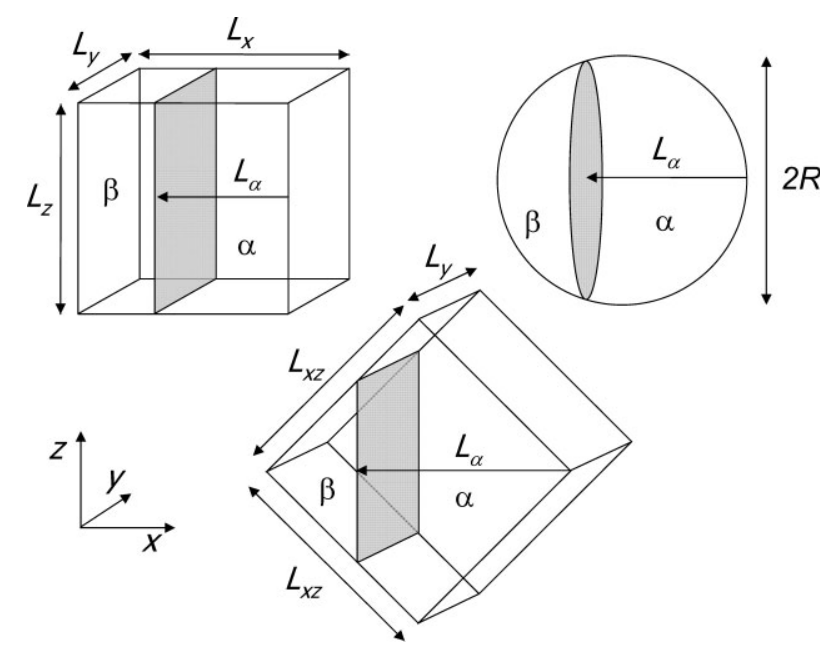

Figure 1. Particle geometries studied, where the gray surface indicates the orientation of the interface between the two phases $\alpha$ and $\beta$. $L_{\alpha}$ determines the position of the interface.

(see Fig. 1), rendering the rectangle equivalent to the diamond, differing only in the relative orientation of the interface. It is illustrative to work out condition (Eq. 5) for these three geometries yielding:

rectangular crystallite

$\Phi_{\alpha}-\Phi_{\beta}+\frac{2 \Omega}{L_{\gamma}} \Delta \sigma_{\gamma}+\frac{2 \Omega}{L_{z}} \Delta \sigma_{z}=0$

spherical crystallite with radius $\mathrm{R}$

$\Phi_{\alpha}-\Phi_{\beta}+\frac{2 \Omega R}{2 R L_{\alpha}-L_{\alpha}^{2}} \Delta \sigma+\frac{2 \Omega\left(R-L_{\alpha}\right)}{2 R L_{\alpha}-L_{\alpha}^{2}} \gamma_{\alpha \beta}=0$

diamond crystallite

$\Phi_{\alpha}-\Phi_{\beta}+\frac{2 \Omega}{L_{\gamma}} \Delta \sigma_{Y}+\frac{\sqrt{2} \Omega}{L_{\alpha}} \Delta \sigma_{x z}+\frac{\Omega}{L_{\alpha}} \gamma_{\alpha \beta}=0$

for $L_{\alpha}<\frac{1}{2} \sqrt{2 L_{x z}}$

$$
\begin{aligned}
& \Phi_{\alpha}-\Phi_{\beta}+\frac{2 \Omega}{L_{\gamma}} \Delta \sigma_{\gamma}+\frac{\sqrt{2} \Omega}{\sqrt{2} L_{x z}-L_{\alpha}} \Delta \sigma_{x z} \\
&+\frac{\Omega}{\sqrt{2} L_{x z}-L_{\alpha}} \gamma_{\alpha \beta}=0 \quad \text { for } \quad L_{\alpha}>\frac{1}{2} \sqrt{2} L_{x z}
\end{aligned}
$$

In these expressions, $L_{\alpha}$ represents the location of the interface between the two coexisting phases as illustrated in Figure 1, and can serve as a metric that plays the same role as the phase fraction of the $\alpha$ phase. $\Delta \sigma_{i}$ for the rectangular and diamond crystallites is the difference in surface energy between $\alpha$ and $\beta$ for the surfaces perpendicular to the side with length $L_{i}$. The dimensions, $R, L_{\alpha}$, $L_{\gamma}, L_{z}$, and $L_{x z}$, are all in terms of the reference crystal, while $\Omega$ corresponds to the volume per interstitial site within the reference crystal (expressing dimensions relative to a reference crystal means they will be unaffected by any volumetric changes of the actual crystallite that may occur as $\mathrm{Li}$ is added to the host). These expressions must be solved together with the equality of chemical potentials condition (Eq. 4).

For the rectangular crystallite, the interface free energy $\gamma_{\alpha \beta}$ does not appear in the equilibrium criterion (Eq. 6), and therefore does not affect the equilibrium concentrations $x_{\alpha}$ and $x_{\beta}$ during two-phase coexistence. This occurs since the interface area in the rectangular crystallite is independent of the phase fraction. Furthermore, the surface area of the $\alpha(\beta)$ phase varies linearly with phase fraction (or equivalently $L_{\alpha}$ for this geometry), rendering the differential change in surface area with $L_{\alpha}$ a constant. As a result, equilibrium criterion (Eq. 6) is also independent of the phase fraction. The equilibrium concentrations $x_{\alpha}$ and $x_{\beta}$ of the coexisting phases, while modified from their values in large crystallites by surface energy differences between $\alpha$ and $\beta$, are therefore independent of the overall $\mathrm{Li}$ concentration within the nanocrystallite. The concentrations $x_{\alpha}$ and $x_{\beta}$ in the spherical and diamond crystallites, in contrast, do depend explicitly on the interface free energy $\gamma_{\alpha \beta}$ as well as the phase fraction. This arises from the fact that the interface area between $\alpha$ and $\beta$ as well as the surface areas in these geometries depend in a nonlinear way on the phase fraction. As a result, the concentrations of coexisting phases, $x_{\alpha}$ and $x_{\beta}$, are no longer constant, but depend on the overall Li concentration within the crystallite.

We assess both the absolute and relative importance of surface and interface free energies on equilibrium compositions by considering $\mathrm{Li}_{x} \mathrm{FePO}_{4}$. While bulk free energies can be obtained by integrating voltage curves for large crystallites, surface and interface free energies are more difficult to measure experimentally. To this end, we use first-principles electronic-structure methods to provide estimates of surface and interface free energies in $\mathrm{Li}_{x} \mathrm{FePO}_{4}$.

i) TEM observations of Chen et al. indicate that the $\alpha / \beta$ interface is mainly perpendicular to the $a$ direction of the orthorhombic $\mathrm{Li}_{x} \mathrm{FePO}_{4}$ unit cell. ${ }^{[19]} \mathrm{Ab}$ initio DFT GGA+U calculations were used to calculate the excess energy associated with a coherent interface in the $b c$-plane in supercells containing periodic regions of $\mathrm{LiFePO}_{4}$ separated by regions of $\mathrm{FePO}_{4}$. Similar values were obtained when fixing the lattice parameters along the $b$ and $c$ directions to their equilibrium values in $\mathrm{LiFePO}_{4}$ and in $\mathrm{FePO}_{4}$, yielding an average of $\gamma_{\alpha \beta}{ }^{y z}=0.96 \mathrm{~J} \mathrm{~m}^{-2}$ (the supercells were allowed to relax in the a-direction).

ii) Surface energies of the Li-poor heterosite phase $(\alpha)$ and Li-rich triphylite phase $(\beta)$ have been calculated by Wang et al. ${ }^{[17]}$ Here, we use values for the surfaces that dominate the $\mathrm{LiFePO}_{4}$ Wulff shape; the [010] surface in vacuum for which GGA+U calculations predict values of $\sigma^{\alpha}=0.24, \sigma^{\beta}=0.64 \mathrm{~J} \mathrm{~m}^{-2}$, and the [201] surface in vacuum for which the same calculations predict $\sigma^{\alpha}=0.77, \sigma^{\beta}=0.52 \mathrm{~J} \mathrm{~m}^{-2}$. For the spherical crystallite, we use an average value of the diamond and rectangle surface energies. The diamond and rectangular crystallites can be viewed as simple approximations to the Wulff shape. ${ }^{[17]}$ Particles obtained by hydrothermal synthesis appear to have aspect ratios $L_{\gamma} / L_{x z}$ 
ranging from $1 / 3$ to $1 / 100^{[19-21]}$ Here, we consider aspect ratios that are fixed $\left(L_{Y}\right)$ $\left.L_{x z}=1 / 3\right)$ and variable.

iii) At room temperature, $\mathrm{Li}_{x} \mathrm{FePO}_{4}$ exhibits a miscibility gap between crystallographically isomorphic host structures with different Li compositions. To accurately parameterize the bulk-free energy of $\mathrm{Li}_{x} \mathrm{FePO}_{4}$ as a function of Li concentration $x$, we use a Riedlich-Kister polynomial expansion together with an idealsolution configurational entropy term (for both Li-vacancy disorder and localized electron disorder). The coefficients of the expansion were fit to reproduce measured open-circuit cell-voltage curves of Meethong et al. ${ }^{[9]}$ Although $\mathrm{Li}_{x} \mathrm{FePO}_{4}$ exhibits strong deviations from thermodynamic ideality as manifested by its composition-temperature phase diagram, ${ }^{[22,23]}$ the ideal solution entropy accounts for the logarithmic dependence of the free energy on Li composition, while the use of the Riedlich-Kister polynomial up to order five enables us to parameterize the deviations of the entropy and enthalpy from ideal solution behavior with high accuracy.

In the following, we investigate the effect of interface free energy and surface free energy on the equilibrium compositions individually.

Figure 2a schematically illustrates how the interface energy penalty leads to a change of the solubility limits. The energy penalty due to the interface energy leads to a reduction of the miscibility gap. Using our first-principles estimate for $\gamma_{\alpha \beta}$ between $\mathrm{LiFePO}_{4}$ and $\mathrm{FePO}_{4}$, and setting all $\Delta \sigma_{i}=0$, the solubility limits are calculated and shown in Figure 2c. The large interface-to-bulk ratio in the sphere leads to the smaller miscibility gap at the same volume compared to the other particle shapes. The impact of particle shape on the equilibrium compositions is further illustrated in Figure 2e, where the angle $\theta$ effectively controls the interface to volume ratio in the diamond shape.

Within these solubility limits, we analyze the equilibrium criteria Equations 4 and 5 (for the rectangle, sphere, and diamond crystallites, respectively). Note that these equilibrium criteria neglect contributions from coherency strains due to differences in lattice parameters between $\alpha$ and $\beta$. Our current analysis only seeks to assess the importance of purely interfacial free-energy terms on equilibrium compositions. The role of coherency strains on equilibrium compositions has been discussed previously, ${ }^{[6,9]}$ while a rigorous derivation of coherency strain equilibrium criteria for $\mathrm{Li}_{x} \mathrm{FePO}_{4}$ will be published elsewhere. Figure $2 \mathrm{~b}$ schematically illustrates the equilibrium criteria Equations 4 and 8 for the diamond crystallite when considering only interface-free-energy contributions. Equation 8 requires that the intercepts of the tangents to $g_{\alpha}$ and $g_{\beta}$ at $x_{\alpha}$ and
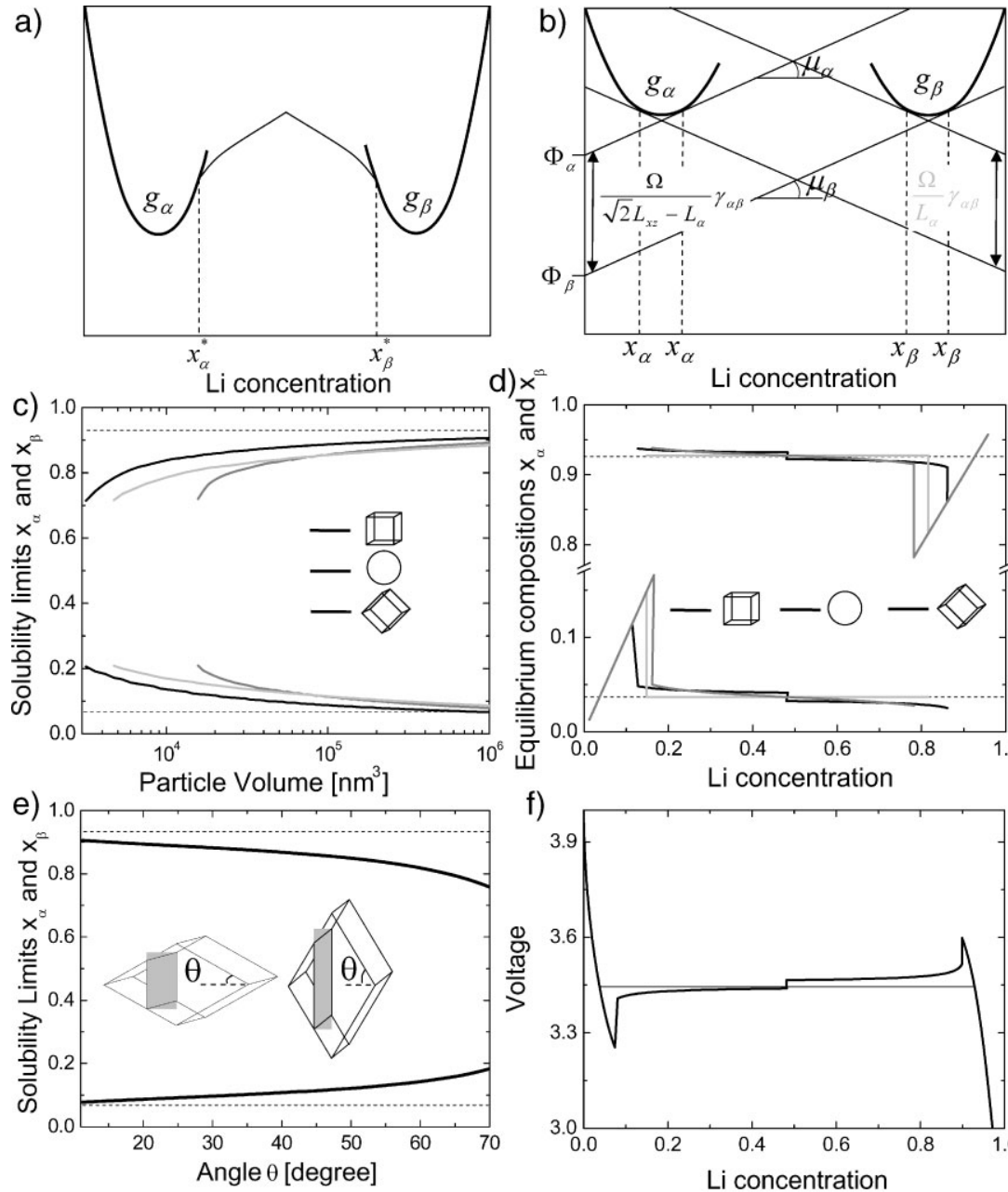

d) $\times$
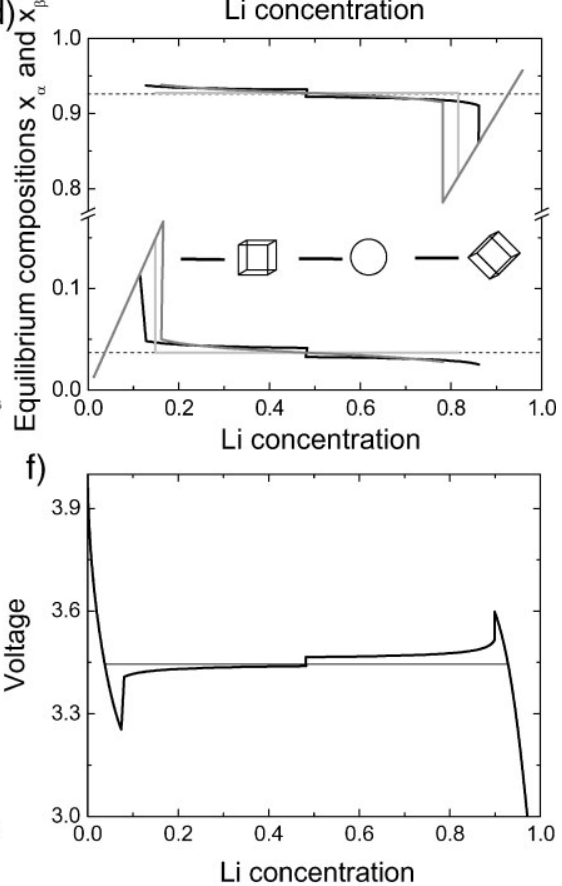

Figure 2. Calculations for two phase coexistence within one particle for non-zero calculated interface energy and zero surface energy, where for the rectangular and diamond shape $L_{y} / L_{x z}=$ 1/3. a) Schematic bulk Gibbs free energy due to the interface energy for the diamond shape, leading to a smaller miscibility gap. b) Schematic bulk Gibbs free energy and common tangent construction for a nanosized diamond-shaped particle to obtain the equilibrium compositions (black: for $\alpha$-phase fraction less than 0.5 , light grey: for $\alpha$-phase fraction greater than 0.5 ). The Gibbs energy is scaled such that the common tangent to the bulk Gibbs energy is horizontal. c) Solubility as a function of particle volume for different particle shapes due to the interface energy. The dotted line indicates the equilibrium compositions in the large particle limit. d) Equilibrium compositions for constant particle volume, $20 \times 10^{3} \mathrm{~nm}^{3}$, as a function of phase fraction for different particle shapes. e) Equilibrium compositions for constant particle volume, $20 \times 10^{3} \mathrm{~nm}^{3}$, as a function of the angle $\theta$ of the diamond shape. f) Voltage curve for a diamond-shaped particle, volume $20 \times 10^{3} \mathrm{~nm}^{3}$, controlling the overall Li concentration.

$x_{\beta}$ with the $x=0$ axis, $\Phi_{\alpha}=g_{\alpha}-x_{\alpha} \mu_{\alpha}$ and $\Phi_{\beta}=g_{\beta}-x_{\beta} \mu_{\beta}$, must differ by $-\Omega \gamma_{\alpha \beta} / L_{\alpha}$ when the phase fraction of the $\alpha$-phase is less that 0.5 . For phase fractions of $\alpha$ greater than 0.5 , the difference of the intercepts $\Phi_{\alpha}=g_{\alpha}-x_{\alpha} \mu_{\alpha}$ and $\Phi_{\beta}=g_{\beta}-x_{\beta} \mu_{\beta}$ with the $x=0$ axis change sign, and must equal to $\Omega \gamma_{\alpha \beta} /\left(\sqrt{2} L_{x z}-L_{\alpha}\right)$.

Figure $2 \mathrm{~d}$ shows the calculated equilibrium compositions as a function of overall composition. Lithiation of $\mathrm{FePO}_{4}$ and delithiation of $\mathrm{LiFePO}_{4}$ initially leads to a solid solution, which is extended due to energy penalty arising from the interface, leading to the reduction of the miscibility gap as shown in Figure 2c. When the two phases coexist, the equilibrium 
compositions $\mathrm{x}_{\alpha}$ and $\mathrm{x}_{\beta}$, as determined by Equations 7 and 8 for the spherical and the diamond crystallites, depend on the overall composition of the crystallite (as a result of the explicit dependence of Eqs. 7 and 8 on $L_{\alpha}$ ). This implies that the composition of both coexisting phases changes during the Li-ion insertion of the electrode material. In contrast, the interface area of the rectangle is independent of the phase fraction, and hence the equilibrium compositions are constant. More generally, this demonstrates that when the size of the interface does not change upon lithiation, the equilibrium compositions are not affected by interface effects. This is clearly an idealized situation, as in reality nucleation of a phase will at least initially lead to a changing interface area.

In Figure 2f, the voltage curve of a diamond-shaped particle, volume $20 \times 10^{3} \mathrm{~nm}^{3}$, is calculated by externally controlling the overall Li concentration, equivalent to a slow (dis)charging experiment. During charging of the electrode (Li insertion) the voltage has to be sufficiently reduced to overcome the energy penalty due to the interface needed for the formation of the two-phase coexistence. Similarly, discharging the electrode will lead to an excess voltage. Because the nucleation barrier will be relatively larger for smaller particles, leading to a smaller miscibility gap in Figure 2c, the excess voltage in Figure $2 \mathrm{f}$ will also be larger. A voltage excess is indeed observed, albeit much smaller, in detailed voltage curves where strain energy is claimed to be the origin. ${ }^{\left[{ }^{9]}\right.}$ Most likely, strain and interface energy have a similar impact. In the present calculations, the excess voltage is most likely strongly overestimated due to the fixed geometry of the interface; in reality, it will depend on detailed nucleation conditions. Because the equilibrium condition (Eq. 8) depends on the overall composition, the single-particle voltage during two-phase coexistence will also depend on the composition. In practice, the electrodes consist of many particles, also having a particle-size distribution leading to a distribution of voltages, ${ }^{[24]}$ averaging out this single particle effect. Note that the discontinuity in Figure $2 \mathrm{~d}$ (and in 2f) near $x=0.5$ is due to the shape of the Gibbs free energy in Figure 2a.

We now investigate the role of differences in surface energies on the characteristics of the two-phase equilibrium within nanocrystallities. As an illustration, we consider two-phase equilibrium within a rectangular crystallite as determined by equilibrium criteria Equations 4 and 6. Equation 4 dictates that the slopes of the tangents to $g_{\alpha}$ and $g_{\beta}$ at $x_{\alpha}$ and $x_{\beta}$, respectively, are equal, while working out Equation 6 stipulates that the intercepts of these tangents with the $x=0$ axis, $\Phi_{\alpha}=g_{\alpha}-x_{\alpha} \mu_{\alpha}$ and $\Phi_{\beta}=g_{\beta}-x_{\beta} \mu_{\beta}$, must differ by $-\left(2 \Omega \Delta \sigma_{y} / L_{y}+2 \Omega \Delta \sigma_{x z} / L_{x z}\right)$. This is schematically illustrated in Figure 3a. For small particle sizes, the difference in surface energy between the two phases can cause a sizable shift in the equilibrium compositions, $x_{\alpha}$ and $x_{\beta}$.
The effect of surface-free-energy contributions is to shift the equilibrium compositions in the same direction relative to those for the bulk. This is illustrated in Figure 3b, showing the impact of particle size on the equilibrium compositions at a phase fraction $\varphi_{\alpha}=0.5$. Figure $3 \mathrm{~b}$ also illustrates the importance of particle shape. Having a minimal surface-to-bulk ratio, the spherical particle shape leads to the smallest shift in equilibrium compositions in Figure 3b. Because the diamond and rectangular shape have the same surface areas at $\varphi_{\alpha}=0.5$, the results are equivalent. The shifts in equilibrium concentrations due to the surface energy are relatively small compared to that due to the interfacial energy (note that in Fig. 2b the scale continues to smaller particle volumes compared to Fig. 3c). It should be noted that the relative magnitude of the surface energies determines whether both $x_{\alpha}$ and $x_{\beta}$ increase $\left(\sigma_{\alpha}<\sigma_{\beta}\right)$ or decrease $\left(\sigma_{\alpha}>\sigma_{\beta}\right)$. The shape dependence of the equilibrium compositions also becomes apparent in Figure $3 \mathrm{c}$, where the aspect ratio of the diamond shape is varied. Effectively, this changes the surfaceto-volume ratio and with it the difference in the average surface energy between both phases $\left(\Delta \sigma=\sigma_{\alpha}-\sigma_{\beta}\right)$. At an aspect ratio $L_{\gamma} /$ $L_{x z}=1.3$, the effective surface energies for the (010) and (201) planes are equal and cancel out, hence the equilibrium compositions remain equal to the bulk values.

A direct consequence of the surface energy entering equilibrium conditions (Eqs. 6-8) is that both the equilibrium conditions and the chemical potential (slope in Fig. 3a) depend on 
the overall composition (for both the spherical and diamond crystallites as a result of the explicit dependence of Equations 6 and 7 on $L_{\alpha}$, not shown) and the size (all shapes). As a result, the voltage for both the spherical and diamond crystallites depends on the overall composition, as shown in Figure 3d. The excess voltage for the various shapes is due to the larger surface energy of the nucleating phase, which depends strongly on particle shape and orientation of the interface. Note that for the spherical particles an average surface energy is used, where $\sigma_{\alpha}<\sigma_{\beta}$, which rationalizes that only excess voltage is required when the $\beta$ phase nucleates in the $\alpha$ phase.

Figure $3 \mathrm{a}$ also indicates that a reduced particle size, using the calculated surface energies ${ }^{[17]}$ in vacuum that imply $\sigma_{\alpha}<\sigma_{\beta}$ using $L_{\gamma} / L_{x z}=1 / 3$, leads to a larger chemical potential, hence a smaller voltage. In reality, the voltage plateau of $\mathrm{LiFePO}_{4}$ appears to increase with decreasing particle size, ${ }^{\left[{ }^{[]}\right.}$indicating effectively $\sigma_{\alpha}>\sigma_{\beta}$. Figure 3c indicates that particle shape may be responsible for this; moreover, the practical surface energies may differ from the calculated values due to the presence of the electrolyte or a surface coating. Recently, we reported ${ }^{[24]}$ in detail on the particle-size-depend voltage, and showed that a distribution of nanoparticle sizes (which is practically always present) leads to a sloped voltage curve consistent with general findings for two-phase nanoinsertion materials. ${ }^{[6,15,16,25]}$

A remarkable finding of the derived equilibrium criteria for phase coexistence within a nanocrystallite is the predicted dependence of the equilibrium compositions on the overall $\mathrm{Li}$ composition of the particle. Consequently, the equilibrium compositions observed by direct structural probes, such as diffraction $\left(x_{\alpha}\right.$ and $\left.x_{\beta}\right)$ and as observed indirectly by electrochemical methods $\left(x_{\alpha}{ }^{*}\right.$ and $\left.x_{\beta}{ }^{*}\right)$, will in general be different. Assuming a slow constant (dis)charge rate, it is well known that the electrochemical potential varies outside the miscibility gap $x_{\alpha}{ }^{*}$ and $x_{\beta}{ }^{*}$. During (dis)charge, the equilibrium compositions are determined at an overall composition that approaches the equilibrium compositions itself. In contrast, direct structural probes such as diffraction can be used to determine the equilibrium compositions $x_{\alpha}$ and $x_{\beta}$ at any overall composition. In the large crystallite limit, where surface and interface contributions are negligible, any variation of the overall $\mathrm{Li}$ composition only changes the relative amount of the coexisting phases, not the compositions within each of the coexisting phases. This picture changes at the nanoscale, where the large contributions from surface and interface free energies to the total free energy renders the Li concentrations within each coexisting phase dependent on the overall Li composition of the crystallite.

Figures $2 \mathrm{c}$ and $3 \mathrm{~b}$ show that solubility limits $x_{\alpha}{ }^{*}$ and $x_{\beta}{ }^{*}$ and equilibrium compositions $x_{\alpha}$ and $x_{\beta}$ change with decreasing particle size, a consequence of the increasing contribution of the interface and surface energy in smaller particles. Recently, a decreased miscibility gap in nanosized $\mathrm{Li}_{x} \mathrm{FePO}_{4}$ has been reported, in most cases based on (dis)charge experiments and changing lattice parameters of the coexisting phases. ${ }^{[5,6]}$ Figure $2 \mathrm{~b}$ indicates that the interface energy may be responsible for such a decreased miscibility gap. Based on open-circuit cell voltages, Meethong et al.$^{[6]}$ report for spherical particles with an average diameter of $34 \mathrm{~nm}$ (volume $\approx 20 \times 10^{3} \mathrm{~nm}^{3}$ ) equilibrium compositions at room temperature of $x_{\alpha} \approx 0.12$ and $x_{\beta} \approx 0.81$. Inspection of Figure $2 \mathrm{c}$ leads, for this particle size, to $x_{\alpha} \approx 0.18$ and $x_{\beta} \approx 0.75$, indicating that the interface energy alone, as calculated at present, could explain the observations. Meethong et al. ${ }^{[9]}$ suggest that strain arising from the coherent coexistence between the $\mathrm{Li}$ rich and Li poor phase is responsible for the change in solubilities, which was formally studied by Cahn et al. ${ }^{[26]}$ While in the present analysis, we have neglected coherency strains, our results indicates that interface free energy plays at least a significant role in affecting the solubility limits in nanosized insertion compounds.

The shift in equilibrium compositions due to the surface energy as seen in Figure $3 \mathrm{~b}$ is remarkably similar to what has been observed in lithiated anatase $\mathrm{TiO}_{2}$ using neutron diffraction. The diffraction results imply that for particle sizes of $40 \mathrm{~nm}$ each particle has a single phase, either the Li-poor (anatase) or the Li-rich (Li-titanate) phase, suggesting that for these sizes the interface energy penalty is too large for two-phase coexistence to occur within the same crystallite. Therefore, the surface energy may be considered as responsible for the experimentally observed increase in equilibrium compositions. Although the calculated shift in Figure 3b (for $\mathrm{Li}_{x} \mathrm{FePO}_{4}$ ) due to the surface energy is in qualitative agreement with the observed shift in lithiated anatase $\mathrm{TiO}_{2}$, the shift reported for the latter is significantly larger $\left(x_{\alpha} \approx 0.2\right.$ at a volume of about $\left.180 \mathrm{~nm}^{3}\right)$, indicating that surface energies have a significant impact on the equilibrium compositions in nanosized anatase $\mathrm{Li}_{x} \mathrm{TiO}_{2}$.

Figure 2e indicates that in addition to particle size, also the particle shape can have a significant impact on the solubility limits for small particle sizes. This is a direct consequence of the changing interface/volume ratio. The miscibility gap decreases for the diamond particle as the angle $\Theta$ is varied to increase the interface/volume ratio. Hence, the present calculations predict differences in equilibrium compositions for similar particle sizes having different shapes. It is noteworthy that the role of surface energy on equilibrium compositions also depends strongly on the particle shape, as illustrated by Figure 3c, where the aspect ratio can even be chosen to yield equilibrium compositions identical to those for the large-crystallite limit.

Our current thermodynamic analysis predicts dependencies of equilibrium compositions on crystallite size and shape that are in qualitative agreement with experimental observations. This indicates that interface and surface energies are significant, if not dominant factors in the description of nanoscale insertion materials. However, in the current description we have neglected several important factors that are also likely to affect equilibrium compositions. Among these is coherency strain energy due to lattice misfit between the coexisting phases. Coherency strain energy is known to narrow the two-phase coexistence regions in a temperature-composition phase diagram. ${ }^{[27]}$ Also the assumption of a fixed interface orientation may lead to an overestimation of the change in solubility limits, because a real system may minimize its interfacial energy by locally changing the interface plane and softening concentration gradients. We have also neglected the increased pressure, $\Delta P$, within the crystallite arising from the surface stress, $f$, equal to the derivative of the surface free energy $\sigma$ with respect to surface strain. The surface stress counters elastic stretching of the surface without creating new surface sites. ${ }^{[28]}$ Other features that become important at the nanoscale include the occurrence of spacecharge effects, which offer additional degrees of freedom for lowering the free energy by releasing the constraint on the local 
charge neutrality near interfaces, while maintaining overall charge neutrality. ${ }^{[1]}$

In our thermodynamic analysis, we first considered two-phase coexistence within a single crystallite, which allowed us to study the impact of the interface energy. However, electrodes containing many particles that can freely exchange Li offer additional degrees of freedom to minimize the overall free energy, whereby two-phase coexistence can also be realized by having a subset of crystallites in the $\alpha$ phase and the remainder in the $\beta$ phase, thereby avoiding the need to introduce costly interfaces between $\alpha$ and $\beta$ within the same crystallite. The transition from intra- to intercrystallite phase coexistence appears to be responsible for the slow voltage relaxation $\left(>10 \mathrm{~h}\right.$ ) in nanosized $\mathrm{Li}_{x} \mathrm{FePO}_{4},{ }^{[29]}$ and in $\mathrm{Li}_{4} \mathrm{Ti}_{5} \mathrm{O}_{12} \cdot{ }^{[30]}$ Such slow relaxation would imply that the intracrystallite approach is in fact a metastable situation in a packed, well-connected nanoparticle electrode. Interestingly, the present results suggest that such a morphological change should have considerable effect on the solubility limits.

Remaining challenges include further refinement of the open-circuit cell potentials of nanomaterials. A number of relevant aspects are currently missing within our free energy model (Eq. 2). First principles calculations by Wang et al., ${ }^{[17]}$ for example, showed that the surface redox potential varies with surface orientation leading to a distribution of potentials. For small particles, a larger fraction of Li-ions will be stored at such surface sites, which will be reflected in the open-circuit cell voltage. This effect can be captured thermodynamically by incorporating surface excess-Li concentrations within the surface free-energy description. The occurrence of space-charge effects at the surface is also likely to affect the local redox-potential.

An analysis of equilibrium criteria derived for two-phase coexistence within a nanocrystallite indicates that surface and interface energies can significantly modify the solubility limits and equilibrium compositions from their values in the bulk. Solubility limits depend strongly on the particle size, but remarkably also the equilibrium compositions depend on the overall composition and particle shape. Such knowledge is vital as it largely determines the functional characteristics when these materials are applied in $\mathrm{H}$-storage devices and Li-ion batteries, for instance. The present calculations for $\mathrm{LiFePO}_{4}$ rationalize recent experimental observations for different particle sizes and shapes.

\section{Acknowledgements}

Financial support from the Netherlands Organization for Scientific Research (NWO) is acknowledged for the CW-VIDI grant of M. Wagemaker. This work is a contribution from the Delft Institute for Sustainable Energy (DISE). A.V.d.V. acknowledges financial support from the petroleum research fund (PRF) of the American Chemical Society (ACS PRF Grant No. 46584-G10). This article is part of the Special Issue on Nanoionics. This article has been amended for print publication.

Received: October 15, 2008

Revised: March 10, 2009

Published online: April 20, 2009

[1] J. Maier, Nat. Mater. 2005, 4, 805.

[2] A. S. Arico, P. Bruce, B. Scrosati, J. M. Tarascon, W. Van Schalkwijk, Nat. Mater. 2005, 4, 366.

[3] J. Maier, Solid State Ionics 2002, 154, 291.

[4] J. Maier, Solid State lonics 2003, 157, 327.

[5] A. Yamada, H. Koizumi, S. I. Nishimura, N. Sonoyama, R. Kanno, M. Yonemura, T. Nakamura, Y. Kobayashi, Nat. Mater. 2006, 5, 357.

[6] N. Meethong, H. Y. S. Huang, W. C. Carter, Y. M. Chiang, Electrochem. Solid-State Lett. 2007, 10, A134.

[7] M. Wagemaker, W. J. H. Borghols, F. M. Mulder, J. Am. Chem. Soc. 2007, $129,4323$.

[8] W. J. H. Borghols, M. Wagemaker, U. Laffont, E. M. Kelder, F. M. Mulder, Chem. Mater. 2008, 20, 2949.

[9] N. Meethong, H. Y. S. Huang, S. A. Speakman, W. C. Carter, Y. M. Chiang, Adv. Funct. Mater. 2007, 17, 1115.

[10] H. G. Schimmel, J. Huot, L. C. Chapon, F. D. Tichelaar, F. M. Mulder, J. Am. Chem. Soc. 2005, 127, 14348.

[11] S. Singh, S. W. H. Eijt, J. Huot, W. A. Kockelmann, M. Wagernaker, F. M Mulder, Acta Mater. 2007, 55, 5549.

[12] C. P. Balde, B. P. C. Hereijgers, J. H. Bitter, K. P. de Jong, J. Am. Chem. Soc. 2008, 130, 6761.

[13] M. Yamauchi, R. Ikeda, H. Kitagawa, M. Takata, J. Phys. Chem. C 2008, 112, 3294.

[14] P. Vermeulen, A. Ledovskikh, D. Danilov, P. H. L. Notten, J. Phys. Chem. B 2006, 110, 20350.

[15] Y. S. Hu, L. Kienle, Y. G. Guo, J. Maier, Adv. Mater. 2006, 18, 1421.

[16] G. Sudant, E. Baudrin, D. Larcher, J. M. Tarascon, J. Mater. Chem. 2005, 15, 1263.

[17] L. Wang, F. Zhou, Y. S. Meng, G. Ceder, Phys. Rev. B 2007, 76.

[18] C. H. P. Lupis, Chemical Thermodynamics of Materials, North-Holland, Amsterdam 1983.

[19] G. Y. Chen, X. Y. Song, T. J. Richardson, Electrochem. Solid-State Lett. 2006, 9, A295.

[20] J. J. Chen, M. S. Whittingham, Electrochem. Commun. 2006, 8, 855.

[21] K. Dokko, S. Koizumi, K. Kanamura, Chem. Lett. 2006, 338.

[22] R. Stevens, J. L. Dodd, M. G. Kresch, R. Yazami, B. Fultz, B. Ellis, L. F. Nazar, J. Phys. Chem. B 2006, 110, 22732.

[23] C. Delacourt, P. Poizot, J. M. Tarascon, C. Masquelier, Nat. Mater. 2005, 4, 254.

[24] M. Wagemaker, A. van der Ven, Electrochem. Commun. 2009, 11, 881.

[25] W. J. H. Borghols, M. Wagemaker, U. Lafont, E. M. Kelder, F. M. Mulder, Chem. Mater. 2008, 20, 2949.

[26] J. W. Cahn, F. Larche, Acta Metall. 1984, 32, 1915.

[27] J. W. Cahn, Acta Metall. 1962, 10, 907.

[28] J. W. Cahn, Acta Metall. 1980, 28.

[29] K.-T. Lee, W. H. Kan, L. F. Nazar, J. Am. Chem. Soc, in press.

[30] M. Wagemaker, E. M. Kelder, J. Schoonman, C. Ringpfeil, U. Haake, D. Lützenkirchen-Hecht, R. Frahm, F. M. Mulder, Adv. Mater. 2006, 18, 3169. 